\title{
Multiple factors affect the regeneration of liver
}

\author{
Gaoxiong Ouyang ${ }^{1}$ \\ Jianyong Liu' (co-first author) \\ Peng Wang ${ }^{2}$ \\ Yuan Ren ${ }^{1}$ \\ Ping $Y{ }^{1}$ \\ Quan Zhou' \\ lun Chen ${ }^{3}$ \\ Bangde Xiang ${ }^{1}$ \\ Yumei Zhang' \\ Zhiming Zhang ${ }^{1}$ \\ Lequn $L i^{1}$
}

\section{SUMMARY}

OBJECTIVE: To study factors affecting the liver regeneration after hepatectomy

METHODS: With 3D reconstitution technology, liver regeneration ability of 117 patients was analysed, and relative factors were studied. RESULTS: There was no statistically difference between the volume of simulated liver resection and the actual liver resection. All livers had different degrees of regeneration after surgery. Age, gender and blood indicators had no impact on liver regeneration, while surgery time, intraoperative blood loss, blood flow blocking time and different ways of liver resection had a significant impact on liver regeneration; In addition, the patients' own pathological status, including, hepatitis and liver fibrosis all had a significant impact on liver regeneration.

CONCLUSION: 3D reconstitution model is a good model to calculate liver volume. Age, gender, blood indicators and biochemistry indicators have no impact on liver regeneration, but surgery indicators and patients' own pathological status have influence on liver regeneration.

KEYWORDS: Liver neoplasms. Carcinoma, hepatocellular. Hepatectomy. Liver regeneration. Imaging, three-dimensional.

\section{INTRODUCTION}

As the third major pathogenic cause of cancer-related death globally ${ }^{1}$, hepatocellular carcinoma (HCC) ranks fifth among the malignant tumour morbidity. Multiple methods have been used for HCC treatments, including surgical resection, radiotherapy and chemotherapy, biological therapy, immunotherapy, etc. Among these different types of treatments, surgical resection functions as the most mainstream method ${ }^{2}$. However, surgical resection would cause impaired or total loss of liver functions, therefore resulting in death of patients postoperatively ${ }^{3}$. HCC patients are reported to show different degrees of hepatitis and fibrosis, which significantly reduced liver functions. Given that liver regeneration 
was relatively low post-surgery, the remained liver volume cannot support the demands of metabolism and maintain homeostasis, therefore leading to impaired liver function, liver failure and the eventually death ${ }^{4}$.

Nowadays, with development of technology, imaging technology matures and computerized simulation surgery come into play. The combination of computer-based automation image software and high-resolution imaging technology has become a new trend to conduct accurate hepatectomy for HCC patients ${ }^{5},{ }^{6}$. Furthermore, using three-dimensional (3D) surgical simulation system, clinicians could evaluate the function of liver and simulate operations before surgery, which significantly reduced the possibility of postoperative liver failure and the potential death of patients. However, impaired liver functions would still occur post-surgery, which might result from the dis-match of the regeneration liver and the body need. Interestingly, little research has been carried out to investigate liver regeneration ability, especially regarding post-surgical liver regeneration ${ }^{7}$. In this study, we hypothesized that factors including age, gender, blood biomarkers, surgical methods, resected liver volume, residual liver volume, surgical margin, hepatitis, liver fibrosis etc., would affect the regeneration rate of liver. Therefore, we analysed liver regeneration rates under different experimental conditions as described below, with the aim to discover better ways to conduct liver surgery.

\section{MATERIAL AND METHODS \\ 2.1 Patients}

117 HCC patients received liver resection surgery were selected (Oct 2012-Oct 2016). Standard experimental procedure was presented in Figure 1A. All patients in this study were in single lesion BCLC-A stage, confirmed of HCC by pathological analysis postoperatively; underwent liver CT scan and enhanced scan (1 week before and 1 week after the surgery); In this study, patients received regular liver resections, at right hemi-hepatic, left hemi-hepatic, left hepatic lobe or right hepatic lobe area respectively. Patients with any of the following situations were excluded: 1) had cholangiocellular carcinoma or metastatic cancer 2) received previous intervention and chemotherapy pre-surgery; 3) had previous liver resection surgery; 4) had diabetes, HIV or other malignant illnesses.
This study was carried out in accordance with the ethical guidelines of the Declaration of Helsinki and approved by the Hospital Ethics Committee of Affiliated Cancer Hospital of Guangxi Medical University. Informed consent was obtained from every patient.

\subsection{Preoperative and postoperative examination}

Patients were examined using 128-slice spiral CT (GE, USA) 1 week before and 1 week after liver resection surgeries. Regular liver and kidney function examination (TBil, ALB, ferritin, ALT, AST, CRE, etc.), coagulation function examination (PT), whole-cell examination (leukocyte, erythrocyte, thrombocyte, etc.), hepatitis B screening (HBsAg, HBsAb; HBeAg, HBeAb; HBcAb), tumour markers detection (AFP) were performed as well.

\subsection{D simulated reconstruction of liver}

The original thin-slice CT scan data was imported into the Myrian XP Liver 3D simulation operation system. The plain, arterial and venous images were selected respectively. Modules including liver, tumour, hepatic vein and portal vein were presented in different colour according to their range and contour (Figure 1B-C). The 3D reconstruction images were generated using the software mentioned above based on the range of the sketch. The simulated liver volume as well as the relative positions of liver, tumour and blood vessels were subsequently calculated and automatically presented.

\subsection{Simulated hepatectomy}

Simulated hepatectomy was performed using Myrian XP Liver software. In brief, the surgical surfaces were determined by the combination of $2 \mathrm{D}$ and 3D images. Simulated resection was strictly performed at surgical surface generated by 3D model in the venous regions, and adjustments were made accordingly using the 2D model. The resected liver volume and the residual liver volume were calculated automatically by Myrian XP Liver software.

\subsection{Calculation of liver regeneration capacity}

The surgery was carried out in accordance with the rules of preoperative liver resection, and the intraoperative and postoperative indicators were collected, including surgery time, intraoperative blood loss, intraoperative blood transfusion, intraoperative hepatic blood occlusion time, postoperative pathological liver inflammation grade and fibrosis stage. The 


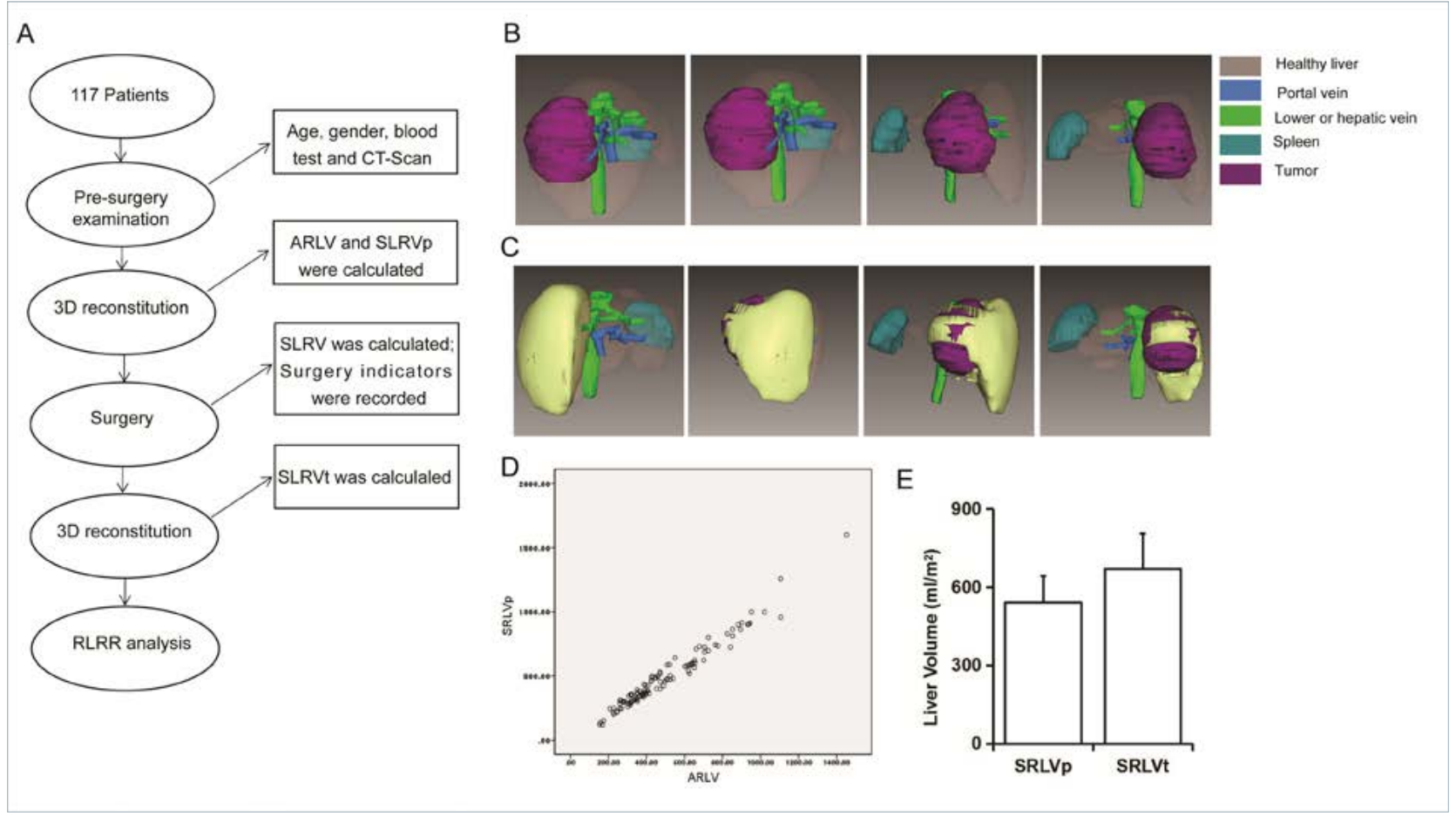

FIGURE 1. 3D reconstruction model is a good model to measure liver volume. (A) Work flow of experimental design and analysis. (B-C) 3D reconstitution of liver and simulated hepatectomy. (D) Correlation analysis of SRLVp and ARLV. (E) Comparison of SRLVp and SRLVt liver volume. Data are shown as means \pm SD. and simulated hepatectomy.

volumes of specimens resected (including tumour tissue and part of normal liver tissue) were measured by drainage method (accurate to $1 \mathrm{ml}$ ). In addition, the volumes of remnant liver before and after hepatectomy were measured using 3D simulation, actual removal liver volume (ARLV) was measured by drainage method. Body surface area (BSA), standard remnant liver volume (SRLV), preoperative expected standard remnant liver volume (SLRVp), postoperative standard remnant liver volume (SLRVt), remnant volume of liver regeneration (RVLR), and remnant liver regeneration rate (RLRR) were calculated as follows:

$$
\begin{gathered}
\operatorname{BSA}\left(\mathrm{m}^{2}\right)=0.0061 \times \text { height }(\mathrm{cm})-0.0128 \times \text { weight }(\mathrm{kg})-0.1529 \\
\operatorname{SRLV}=\operatorname{RLV}(\mathrm{ml}) / \operatorname{BSA}\left(\mathrm{m}^{2}\right) \\
\operatorname{SLRVp}=\operatorname{RLVp}(\mathrm{ml}) / \operatorname{BSA}\left(\mathrm{m}^{2}\right) \\
\text { SLRVt }=\operatorname{RLVt}(\mathrm{ml}) / \operatorname{BSA}\left(\mathrm{m}^{2}\right) \\
\operatorname{RVLR}=\operatorname{SLRVt}-\operatorname{SLRVp} \\
\text { RLRR }=\text { RVLR } / \operatorname{SRLVp}=(\operatorname{SLRVt}-\operatorname{SLRVp}){ }^{*} 100 \% / \operatorname{SLRVp}
\end{gathered}
$$

\subsection{Statistical analysis}

The statistical analysis was performed using SPSS 22.0 to calculate mean and standard error. Paired two sample t-test was used for resected liver volume, SRLVt and STLVp analysis, and unpaired two-sample t-test was used for RLRR analysis. One-way analy- sis of variance (ANOVA) and post-hoc analysis (LSD) were performed as indicated after confirming the homogeneity of variance. Correlation analysis between SRLVt and SRLVp was carried out using Pearson analysis. $P$ value $<0.05$ was considered as statistically significant in this study $\left({ }^{*} \mathrm{p}<0.05,{ }^{* *} \mathrm{p}<0.01,{ }^{* * *} \mathrm{p}<0.005\right)$.

\section{RESULTS}

\subsection{Patients}

Patients' information was presented in Table 1. There were 98 male patients (83.76\%) and 19 female patients $(16.24 \%)$ with mean age at $\sim 50$ years old (ranging from 24-83 years old). Among all the 117 patients, 96 showed HBsAg positive. According to preoperative analysis, there were 111 A-stage (94.87\%) and 6 B-stage patients (5.13\%). Average tumour size in this study was $5.35 \mathrm{~cm}$ (ranging from $3.60 \mathrm{~cm}$ $14.10 \mathrm{~cm})$.

19 patients received right hemi-hepatic resection (16.24\%), 21 received left hemi-hepatic resection (17.95\%), 21 received left hepatic lobe resection (17.95\%) and 56 received right hepatic lobe resection (47.86\%). Average time of surgery time was 190s (ranging from 90s-370s), average blood loss was 200ml (ranging from 50ml-2100ml), average blood 
flow blockage time was 20mins (ranging from Omin42mins).

Patients were rated into GO (15 patients, $12.82 \%)$, G1 (34 patients, 29.06\%), G2 (50 patients, 42.74\%) and G3 (18 patients, 15.38\%) in terms of hepatitis B stages. Based on the degree of liver fibrosis, patients were rated into SO (14 patients, 11.97\%), S1-2 (35 patients, 29.91\%), S3 (42 patients, 35.90\%) and S4 (26 patients, $22.22 \%)$ stages.

3.2 3D reconstruction model is a good model to measure liver volume

Measuring the volume of liver is a challenging area in liver research. Recently, people have developed 3D reconstitution method to measure liver volume; however, it is not always accurate. To measure the regeneration rate of liver, we used the Myrian XP Liver software combined with thin-slice CT scan, and $3 \mathrm{D}$ reconstitution was generated (Figures $1 \mathrm{~B}$ and 1C). ASLV, SLRVp and SLRVt were measured (Figure 1A). Results showed that ASLV and SLRVp have a linear relationship (Figure 1D), and no difference was observed between ASLV and SLRVp, indicating that predicated removal liver volume is not different from the real volume of liver resection. Thus, 3D reconstitution is a good model to measure liver volume.

\subsection{Comparison between preoperative and} postoperative liver volumes

To investigate if residual liver regenerated after resection, we compared the liver volume preoperatively and postoperatively using experiments and 3D simulation.

SRLVt was $670.31 \mathrm{ml} / \mathrm{m}^{2}$, and SRLVp was $541.60 \mathrm{ml} /$ $\mathrm{m}^{2}$. Statistical analysis confirmed there was significant difference between SRLVt and SRLVp ( $\mathrm{t}=-26.17$ $-{ }^{*} \mathrm{p}<0.05$ ) (Figure 1E), suggesting that the liver regenerated significantly post-surgery. RVLR was $128.71 \mathrm{ml} / \mathrm{m}^{2}$, and the RLRR was $23.73 \%$ in this study.

\subsection{Analysis of factors affecting liver regener- ation}

In this section, factors affecting liver regeneration were analysed respectively.

Our results showed that several factors exhibited none-significant impacts on liver regeneration, including age, gender, leukocyte number, erythrocyte number, ALB, ALT, TBil, AFT, PT, HBsAg, serum CRE and Child-Pugh class. These factors should not be taken into consideration for liver resection. In this section, we investigated the effects of blood loss amount, surgical methods, liver blood blocking time, surgery time, hepatitis and liver fibrosis on liver regeneration ability.

\subsubsection{Effects of surgical conditions}

\subsubsection{Effects of blood loss on RLRR}

To investigate if blood loss affected liver regeneration, we compared RLRR of patient group A (lost $>800 \mathrm{ml}$ during surgery) to that of patient group B (lost $<800 \mathrm{ml}$ ) (Figure 2A). RLRR from patient group B (27.11\%) was significantly higher than RLRR from group A $(15.12 \%)\left(t=8.459-{ }^{* * *} \mathrm{p}<0.001\right)$, suggesting blood loss inhibited postoperative live regeneration.

\subsubsection{Effects of liver blood blocking time on RLRR}

With the purpose of investigating if liver blood blocking time affected liver regeneration, we compared the RLRR of patient group C (total blocking time $>30$ mins) to that of patient group $\mathrm{D}$ (total blocking time $<30 \mathrm{mins}$ ) (Figure 2B). Statistical analysis demonstrated that RLRR from group C (17.48\%) was significantly lower than that of group D (25.34\%) $(\mathrm{t}=4.191$, ${ }^{* * *} \mathrm{p}<0.001$ ), indicating that less liver blood blocking time would contribute to liver regeneration process.

\subsubsection{Effects of surgery time on RLRR}

To investigate if the surgery time affected liver regeneration, we compared the RLRR of patients group

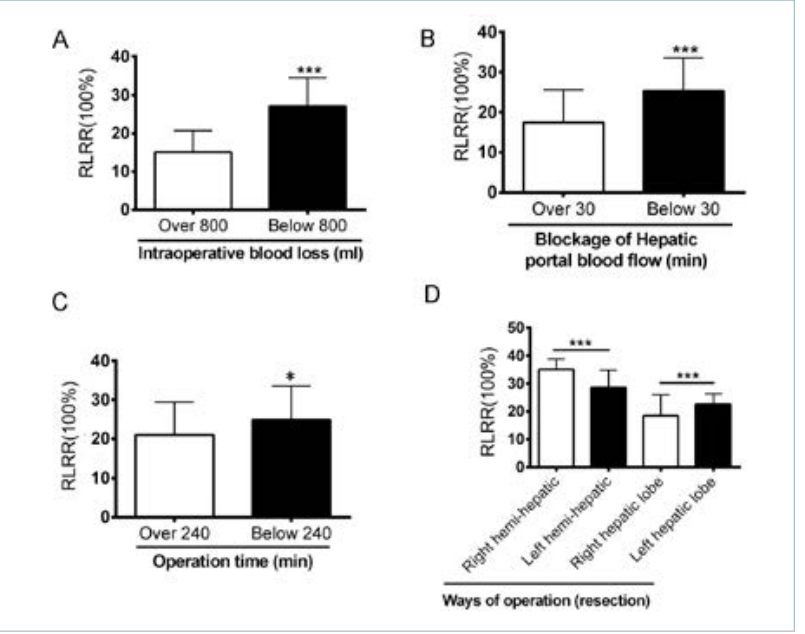

FIGURE 2. Effects of operation conditions on liver regeneration rate. Quantification of liver regeneration rate under the treatment with different blood loss amount $(A)$, blockage time (B), operation time (C) and area of resections (D). Statistical analysis was performed as described in Material and Methods (student t-test, Error bar: $\pm \mathrm{SD} ;{ }^{*} \mathrm{p}<0.05,{ }^{\star \star *} \mathrm{p}<0.005$ ) 
$\mathrm{E}$ (> 240mins) and that of patient group F (<240mins) (Figure 2C). RLRR from group F (24.89\%) was significantly higher than that of group $\mathrm{E}(21.02 \%)(\mathrm{t}=2.225$, $\left.{ }^{*} p=0.028\right)$, suggesting less surgery time showed a positive effect on liver regeneration.

\subsubsection{Effects of surgical methods}

Furthermore, we investigated the effects of resected regions on liver regeneration rate. As shown in Figure 2D, the RLRR of patients that received right hemi-hepatic resection was $35.07 \%$ (group G), that of patient who received left hemi-hepatic resection was 28.62\% (group H), that of patients that received left hepatic lobe resection was $22.60 \%$ (group I), and that of patients that received right hepatic lobe resection was $18.47 \%$ (group J) respectively. One-way ANOVA and post-hoc analysis confirmed that there were significant difference between the RLRR of each group $\left(\mathrm{F}=38.92,{ }^{* * *} \mathrm{p}<0.001\right)$, indicating that patients who received right hemi-hepatic resection showed the highest liver regeneration rate.

\subsubsection{Effects of hepatitis and liver fibrosis}

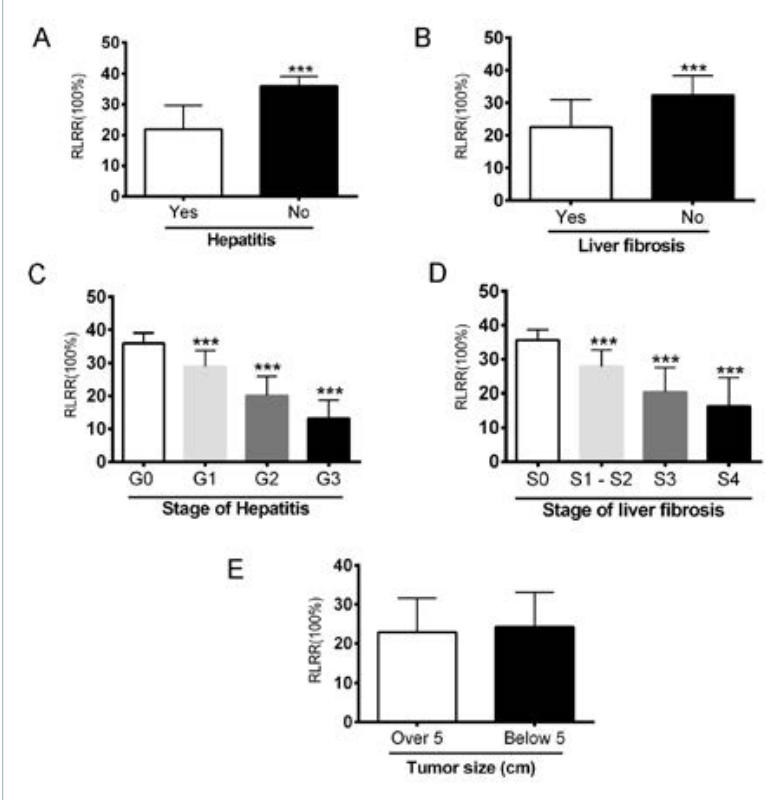

FIGURE 3. Effects of previous liver conditions on liver regeneration rate. Quantification of liver regeneration rate of patients with/without hepatitis (A) as well as that of patients with/without liver fibrosis (B). Patients at different hepatitis stages (C) or different fibrosis stages (D) were subsequently compared. Size of tumor was analyzed (E) as well. Statistical analysis was performed as stated in Material and Methods (student t-test). Multi-group comparisons of the means were carried out by one-way ANOVA with post-hoc contrasts by Levene. Statistical significance was set at $p<0.05$. (Error bar: \pm SD; $\left.{ }^{* \star *} \mathrm{p}<0.005\right)$.
In addition, pathology status of liver was reported to affect liver regeneration postoperatively. Here we analysed the influence of hepatitis and fibrosis on liver regeneration ability. We analysed the RLRR of patients with (group J) / without hepatitis (group $\mathrm{K}$ ) as well as the RLRR of patients with (group L) / without liver fibrosis (group M). According to Figure 3A-B, statistical analysis showed that there were significant differences between group $\mathrm{J}(21.92 \%)$ and $\mathrm{K}(36.0 \%)\left({ }^{* * *} \mathrm{p}<0.001\right)$ as well as between group $\mathrm{L}$ $(22.56 \%)$ and $\mathrm{M}(32.37 \%)\left({ }^{* * *} \mathrm{p}<0.001\right)$, suggesting that patients with previous liver pathogenetic conditions exhibited decreased liver regeneration ability.

Moreover, patients with hepatitis or liver fibrosis were further analysed according to the classification stages respectively. RLRR of patients in GO, G1, G2 and G3 stage were shown in Figure 3C. One-way ANOVA and post-hoc LSD analysis confirmed there were significant differences between each group $(\mathrm{F}=$ $\left.71.39,{ }^{* * * *} \mathrm{p}<0.001\right)$. Similarly, RLRR of patients in SO, S1-2, S3 and S4 stage (Figure 3D) showed significant difference between each group $\left(\mathrm{F}=26.11,{ }^{* * *} \mathrm{p}<0.001\right)$, suggesting that decreased liver regeneration rate of patients with hepatitis or liver fibrosis were associated with the disease development stages.

\section{DISCUSSION}

With the fast development of imaging technology, multi-slice spiral CT and 3D reconstruction technology have been increasingly used in clinical practice ${ }^{8}$. Due to cross and integration of clinical medicine, imaging, pathophysiology and computer science, virtual liver visualization and preoperative simulated hepatectomy have become available?

In hepatectomy, the resection scope varied depending on the location of tumour and the severity of complicated liver cirrhosis, resulting in inconsistences between the theoretical resection line and the actual resection line. In addition, surgical margins varied due to different surgical areas. Therefore, to reduce interference by human factors, it is important to make the simulated surgical resection close to actual liver resection as possible.

In this study, we selected patients with regular liver resection for the following reasons: 1) range of regular liver resection was fixed, 2) preoperative simulation could be easily measured, intraoperative surgery could be performed according to the typical pipelines, 3) preoperative simulated resection is co- 
incident with the actual resected liver volume. All of these can reflect the postoperative residual liver regeneration capacity objectively and accurately. Myrian XP Liver software was applied to perform preoperative simulated hepatectomy. There was no significant difference between ARLV-calculated and SRLVp-simulated liver volume $(\mathrm{p}=0.15)$, further analysis showed that there was positive correlation between ARLV simulation and SRLVp calculation $\left({ }^{* * *} \mathrm{p}\right.$ $<0.001)$ indicating that the results of the computerized simulation could be used as a relatively accurate reference for liver volume measurement in clinical applications ${ }^{10,11 .}$

Hepatectomy has been reported as the most effective way of liver cancer treatment. To meet the requirement of metabolism, postoperative residual liver showed different degrees of proliferation under various conditions. We reported that postoperative residual livers regenerated to various degrees in this study, which is consistent with previous research ${ }^{12}$.

Further analysis regarding liver regeneration showed that surgery time, intraoperative blood loss and hepatic blood flow blockage time were significantly correlated with residual liver regeneration $\left.{ }^{* * * *} \mathrm{p}<0.001\right)$. Long surgery time, severe blood loss and long blockage time would increase the difficulty of the surgery. Affecting factors including deep tumour location, heavy adhesion with surrounding tissues, and the broken blood vessels caused by the surgery, resulted in extensive intraoperative bleeding, intraoperative ischemia-reperfusion injury, thus leading to decreased production of liver regeneration factors ${ }^{13}$, which decelerated the rate of proliferation of postoperative residual livers ${ }^{14}$, and ultimately reduced hepatocyte regeneration. Interestingly, data obtained in this study suggested that ages and HBsAg did not affect liver regeneration significantly. Theoretically, younger patients should have the highest regeneration rate. HBsAg-positive patients should have relatively high regeneration rate due to compensatory effect. However, in this study, there were no significant differences between older patients (> 60 years old) and younger patients ( $\leq 60$ years old), as well as between HBsAg positive and HBsAg negative patients. We speculated that the reason for this might be the limited observation time, which is not long enough to detect the effects of age and HBsAg infection on liver regeneration rate, which should be further investigated in the future.
In this study, the right hemi-hepatic resection resulted in the highest liver regeneration rate, which might be due to the highest liver volume that was removed. We observed that in patients who received right hemi-hepatic resection, there were insufficient amount of functional liver cells to maintain homeostasis, therefore the liver function did not meet requirements of normal metabolism, which urgently stimulated the fast regeneration of the liver. In addition, high amount of resection volume gave space for liver regeneration, which could also stimulate the rapid growth of liver ${ }^{15}$. Compared to patients discussed above, patients who received left hemi-hepatic, left hepatic lobe or right hepatic lobe resection remained with a relatively functional liver that could meet the demand of liver homeostasis, which gave rise to low rate of postoperative regeneration. Data collected in this section suggested ability of residual liver to maintain metabolic homeostasis determined liver regeneration rates.

In addition, we found that stages of liver fibrosis and liver regeneration rate were negatively correlated. We speculated that liver fibrosis might induce liver cells undergo necrosis, and this resulting in decreased regeneration rate. Furthermore, we found that compared to patients did not have hepatitis and fibrosis, patients with hepatitis and liver fibrosis exhibited decreased liver regeneration rate, which is opposite to previous reports ${ }^{16,17}$. The possible reason for this was that self-repair ability of liver or that the short observation time after hepatectomy was not enough to detect significant difference, which should also be investigated in the future study.

Collectively, the liver regenerated to varying degrees post-hepatectomy. We found that rates of liver regeneration were affected by several factors, including surgery time, amount of intraoperative blood loss, blockage time of intraoperative liver blood, surgical methods, inflammation and liver fibrosis stages. In this study, patients with highest liver regeneration rate after hepatectomy showed preoperative low degrees of liver inflammation and fibrosis, they received right hemi-hepatic resection, and were treated with shorter surgery time, less intraoperative blood loss, shorter liver blood flow blocking time.

As a hot topic in research and clinical application, ALPPS surgery is known as stage II hepatectomy: Stage I is to perform transection of the liver along the falciform ligament, right portal vein 
ligation and resection of gallbladder. Stage II is to perform extended right hepatectomy when residual liver volume was enough ${ }^{18-20}$. One week after surgery, the rate of residual liver regeneration was $74 \% \sim 87 \%$, which was much higher than that of this study. The livers of patients who received ALPPS were obviously regenerated in a shorter term. This high regeneration rate is speculated to be caused by the remaining arterial blood supply at the cancerous area, therefore supporting the metabolism as the temporary liver. Meanwhile large amount of portal vein blood flowed into the remaining liver, which promoted the rapid proliferation of the liver. But stage II hepatectomy should be carried out on the basis of the recovery of stage I hepatectomy ${ }^{20}$, and the regeneration rate in our stage I hepatectomy was not high enough to perform stage II hepatectomy. In this study, patients showed local inflammatory response postoperatively. Under the condition of extensive resected area, liver regeneration rate of the patients who received liver resection was significantly less than that of patients that received ALPPS. Therefore, we believed the un-removed tumour tissue promoted liver regeneration, which might be due to the growth factors secreted by tumour tissue, but this hypothesis needs to be further investigated.

\section{CONCLUSION}

This study investigated the liver regeneration ability and the relevant affecting factors, providing scientific support and guidance for preoperative plan, intraoperative operation and postoperative prediction, therefore shedding lights on new clues aiming to improve liver regeneration ability as well as postoperative recovery rate for future clinical practice.

\section{ACKNOWLEDGEMENTS}

This work was supported by Medical and Health Suitable Technology Research and Development Project of Guangxi (Grant No. S201516) and Guangxi Scientific Research and Technology Development Plan (Grant No. 1355005-3-3).

\section{Funding}

This work was supported by Medical and Health Suitable Technology Research and Development Projects of Guangxi (grant S201516) and Guangxi Scientific Research and Technology Development Plan (grant 1355005-3-3)

\section{Conflicts of interest}

The authors declare no conflicts of interest.

\section{RESUMO}

OBJETIVO: Estudar os fatores que afetam a regeneração hepática após hepatectomia.

MÉTODOS: A capacidade de regeneração hepática de 117 pacientes foi analisada com a tecnologia de reconstituição 3D e foram estudados os fatores relacionados.

RESULTADOS: Não houve diferença estatística significante entre o volume de ressecção hepática simulada e a ressecção atual. Todos os fígados apresentaram diferentes graus de regeneração após cirurgia. Idade, gênero e indicadores sanguíneos não tiveram impacto na regeneração hepática, enquanto que tempo de cirurgia, perda sanguínea intraoperatória, tempo de bloqueio do fluxo sanguíneo e diferentes formas de ressecção mostraram impacto significante na regeneração do órgão. Além disso, condições patológicas dos pacientes, incluindo hepatite e fibrose hepática, tiveram impacto significante na regeneração hepática.

CONCLUSÃo: O modelo de reconstituição 3D é um bom modelo para calcular o volume do fígado. Idade, gênero, indicadores sanguíneos e bioquímicos não tiveram impacto na regeneração hepática, mas indicadores operatórios e condição patológica dos pacientes mostraram influência na regeneração do órgão.

PALAVRAS-CHAVE: Neoplasias hepáticas. Carcinoma hepatocelular. Hepatectomia. Regeneração hepática. Imagem tridimensional.

\section{REFERENCES}

1. Block TM, Mehta AS, Fimmel C), Jordan R. Molecular viral oncology of hepatocellular carcinoma. Oncogene. 2003;22(33):5093-107.

2. Glantzounis GK, Tokidis E, Basourakos SP, Ntzani EE, Lianos GD, Pentheroudakis $G$. The role of portal vein embolization in the surgical management of primary hepatobiliary cancers. A systematic review. Eur I Surg Oncol. 2017;43(1):32-41.

3. Allard MA, Adam R, Bucur PO, Termos S, Cunha AS, Bismuth H, et al. Posthepatectomy portal vein pressure predicts liver failure and mortality after major liver resection on noncirrhotic liver. Ann Surg. 2013;258(5):822-9.

4. Ladenstein R, Pötschger U, Pearson AD, Brock P, Luksch R, Castel V, et al. Busulfan and melphalan versus carboplatin, etoposide, and melphalan as high-dose chemotherapy for high-risk neuroblastoma (HR-NBL1/ SIOPEN): an international, randomised, multi-arm, open-label, phase 3 trial. Lancet Oncol. 2017;18(4):500-14.

5. Aoki T, Murakami M, Koizumi T, Kusano T, Fujimori A, Enami Y, 
et al. Preoperative tattooing for precise and expedient localization of landmark in laparoscopic liver resection. J Am Coll Surg. 2015;221(5):e97-e101.

6. Chen XP, Zhang WD, Wang D, Cui W, Yu YL. Image classification of liver cancer surrounding right hepatic pedicle and its guide to precise liver resection. Int J Clin Exp Med. 2015;8(7):11093-100.

7. Nagino M, Ando M, Kamiya J, Uesaka K, Sano T, Nimura Y. Liver regeneration after major hepatectomy for biliary cancer. Brit I Surg. 2001;88(8):1084-91.

8. Maher MM, Kalra MK, Sahani DV, Perumpillichira IJ, Rizzo S, Saini S, et al. Techniques, clinical applications and limitations of 3D reconstruction in CT of the abdomen. Korean / Radiol. 2004;5(1):55-67.

9. Saito S, Yamanaka J, Miura K, Nakao N, Nagao T, Sugimoto T, et al. A nove 3D hepatectomy simulation based on liver circulation: application to liver resection and transplantation. Hepatology. 2005;41(6):1297-304.

10. Hiroshige S, Shimada M, Harada N, Shiotani S, Ninomiya M, Minagawa $R$, et al. Accurate preoperative estimation of liver-graft volumetry using three-dimensional computed tomography. Transplantation. 2003;75(9):1561-4

11. Wigmore S), Redhead DN, Yan XJ, Casey J, Madhavan $\mathrm{K}$, Dejong CH, et al Virtual hepatic resection using three-dimensional reconstruction of helical computed tomography angioportograms. Ann Surg. 2001;233(2):221-6.

12. Nagasue N, Yukaya H, Ogawa Y, Kohno H, Nakamura T. Human liver regeneration after major hepatic resection. A study of normal liver and livers with chronic hepatitis and cirrhosis. Ann Surg. 1987;206(1):30-9.
13. Cressman DE, Greenbaum LE, DeAngelis RA, Ciliberto G, Furth EE, Poli $V$, et al. Liver failure and defective hepatocyte regeneration in interleukin-6-deficient mice. Science. 1996;274(5291):1379-83.

14. Shi $\mathrm{JH}$, Huitfeldt HS, Suo ZH, Line PD. Growth of hepatocellular carcinoma in the regenerating liver. Liver Transpl. 2011;17(7):866-74.

15. Gruttadauria S, Parikh V, Pagano D, Tuzzolino F, Cintorino D, Miraglia $\mathrm{R}$, et al. Early regeneration of the remnant liver volume after right hepatectomy for living donation: a multiple regression analysis. Liver Transpl. 2012;18(8):907-13.

16. Dorn C, Heilmann J, Hellerbrand C. Protective effect of xanthohumo on toxin-induced liver inflammation and fibrosis. Int | Clin Exp Pathol. 2012;5(1):29-36

17. Chan CC, Cheng LY, Lin CL, Huang YH, Lin HC, Lee FY. The protective role of natural phytoalexin resveratrol on inflammation, fibrosis and regeneration in cholestatic liver injury. Mol Nutr Food Res. 2011;55(12):1841-9.

18. Petrowsky $H$, Györi G, Oliveira M, Lesurtel M, Clavien PA. Is partial-ALPPS safer than ALPPS? A single-center experience. Ann Surg. 2015;261(4):e90-2.

19. Vivarelli $M$, Vincenzi $P$, Montalti R, Fava G, Tavio $M$, Coletta $M$, et al ALPPS procedure for extended liver resections: a single centre experience and a systematic review. PloS One. 2015;10(12):e0144019.

20. Brustia R, Scatton O, Perdigao F, El-Mouhadi S, Cauchy F, Soubrane O. Vessel identifications tags for open or laparoscopic associating liver partition and portal vein ligation for staged hepatectomy. J Am Coll Surg. 2013;217(6):e51-5. 